\title{
Mining Express Service Innovation Opportunity From Online Reviews
}

\author{
Ning Zhang, Qingdao University, Qingdao, China \\ Rui Zhang, Qingdao University, Qingdao, China \\ Zhiliang Pang, Qingdao University, Qingdao, China \\ Xue Liu, Qingdao University, Qingdao, China \\ Wenfei Zhao, Qingdao University, Qingdao, China
}

\begin{abstract}
In order to further meet the diversified needs of customers and enhance market competitiveness, it is necessary for express delivery enterprises to carry out service innovation. From the perspective of customer demand, this paper proposes a framework for mining service innovation opportunities. This framework uses text mining to analyze user-generated content and tries to provide a scientific service innovation scheme for express enterprises. Firstly, the authors crawl online reviews of express companies and use LDA model to identify service attributes. Secondly, customer satisfaction is calculated by sentiment analysis, and simultaneously, the importance of each service attribute is calculated. Finally, the authors carry out an opportunity algorithm with the results of text mining to quantify the innovation opportunities of service attributes. The results show that the framework can effectively identify service innovation opportunities from the perspective of customer demand. This study provides a new way to explore the direction of service innovation from the perspective of customer demand.
\end{abstract}

\section{KEYWORDS}

Express Service, Online Reviews, Opportunity Algorithm, Service Innovation, Text Mining

\section{INTRODUCTION}

In recent years, with the development of e-commerce, customer demands for express service are not just restricted to the concept of 'fast'. From the perspective of customers, express service is an important part of e-commerce. Goods are delivered to the customers from different places by express, and then e-commerce can be realized. The development of express service can greatly promote the development and optimization of e-commerce, even cross-border e-commerce. With the application of new technologies, express service is developing vigorously. At the same time, the demands of customers have gradually become diversified. In order to meet the diversified needs of customers, both academia and business are making efforts. Scholars have studied the innovations of express service from the perspective of finance or algorithm to promote the development of e-commerce (Li et al., 2020; Feng, 2018). Many express companies also continue to introduce innovative mode to optimize the customer experience of express service, for instance, SF EXPRESS launched "HIVE BOX" which 
is a kind of intelligent express cabinet to solve the end delivery problem. Also, CAINIAO, a company subordinate to Alibaba Group, launched unmanned vehicles for short-distance delivery. However, it is not clear whether these innovations work for customers. So, what innovations can meet customer needs and achieve results is worth exploring.

Traditional express service innovation usually starts from within an enterprise. Enterprises carry out logistics innovation from the perspective of dynamic capability (Chen et al., 2019) and service maturity (Liu et al., 2019), etc. Service innovation from the perspective of customers often develops slowly due to the lack of data and the difficulties in collecting data. With the development of the Internet, customers have provided a significant number of online reviews on social media platforms. These serve as customer feedback and become a rich data source for enterprises (He et al., 2016; Pournarakis et al., 2017; Xiao et al., 2016). However, few scholars have studied express service innovation using online reviews from the perspective of customers, although customer demands are the behavioral basis of express service innovation. Express service innovation based on online reviews is not only an opportunity to meet customer needs, but also a big challenge.

Based on above, this paper proposes to carry out service innovation with online reviews from the perspective of customers. A framework is constructed for mining service innovation opportunities, which performs text mining to automatically extract service attributes, and adopt an opportunity algorithm to quantify the innovation opportunities in service attributes. It is seen that the combination of text mining and opportunity algorithm can effectively mine service innovation opportunities from the perspective of customer demand, which can provide new ideas for the direction of express service innovation and also promote the management and optimization of AI- powered e-commerce.

The organization of this paper is as follows. Section II discusses theoretical background. Section III describes the proposed service innovation opportunity mining framework. Section IV provides the results and discussion of the experiment. Section V concludes this paper.

\section{BACKGROUND}

\subsection{Service Innovation and Opportunity Algorithm}

The innovations in the service industry are essentially different from that in other industries, especially the production industry, which has been researched considerably. The main reason is that the product form of the service industry is more diversified. Researchers have different perspectives on service innovation. Gallouj (1998) considered that the reverse product life cycle model proposed by Barras in the 1990s was the symbolic beginning of service innovation from the perspective of the product service cycle. Later, research scholars began to consider that the main purpose of service innovation is to meet customer needs. Some scholars proposed that enterprises can carry out service innovation from the perspective of service technology development (Sundbo, 2008; Xu et al., 2020a; Xu et al., $2020 \mathrm{~b}$ ), and that they could meet customer needs by improving the service products and delivery process. Paton and McLaughlin (2008) defined the connotation of service innovation from the perspective of economic development, technological change, and research theory.

The opportunity algorithm was proposed by Ulwick (2005). It can measure whether to meet the needs of customers by using two factors, namely importance and customer satisfaction. From the perspective of result-driven innovation, opportunity algorithm is customer-oriented and forms the concept of service innovation from the perspective of customers. This concept holds that when demand is important but not well met, there will be opportunities for innovation; as customer demand becomes more important and customer satisfaction decreases, opportunities for value creation will also increase. As a customer-oriented innovation opportunity measurement method, many scholars apply it to the research of planning product or service innovation. Killen et al. (2005) provided suggestions for enterprises to design innovative and optimal products by identifying customer needs with the greatest opportunity value. Opportunity algorithm is also used to find unmet customer needs, 
which can measure the competitive advantage of enterprises and find the direction for improvement (Hinterhuber, 2013), and also can derive customer satisfaction and target management structure (Yen et al., 2007).

Most of the existing researches on opportunity algorithm use questionnaire to collect data. However, due to the inherent limitations of questionnaires, there are often problems of insufficient data recovery and high collection costs (Culotta and Cutler, 2016; Groves, 2006). With the development of the Internet, online word-of-mouth (as the carrier of customer demand and customer satisfaction) has become the focus of scholars and enterprises. Jeong et al. (2019) chose online reviews as the data source, and used opportunity algorithm to identify the opportunity value and improvement direction of products. All information is mined from online reviews, which is a new practice for opportunity algorithm. Based on above, we can see that the opportunity algorithm can provide appropriate solutions for product or service innovation and improvement. So, as an important part of e-commerce, express service needs to measure its innovation opportunities to improve customer experience. With the help of online reviews and text mining technology for data processing, the opportunity algorithm can be constructed to provide a new perspective for express service innovation. This work can be conducive to further promote the optimization and management of e-commerce.

\subsection{Attribute Extraction and Topic Model}

The extraction of service attributes and their keywords is the important step of service innovation opportunity mining. At present, most researches extract attributes and their keywords by constructing a domain ontology (Liu et al., 2012; Zhang et al., 2012). This method extracts attributes by obtaining an expression for product or service attributes and their relationships. However, this method cannot dig out potential service attributes from the perspective of customers. In addition, some scholars use text clustering methods to extract product or service attributes (Abualigah et al., 2017; Kushwaha and Pant, 2018). However, in traditional text clustering algorithms, many parameters of the model are generally determined in advance, and it is difficult to explore the hidden topic distribution from a large number of word vector spaces. With the development of machine learning, the topic model, which randomly estimates parameters in the statistical form of probability distribution, is capable of mining potential topics from a large amount of text. Latent Dirichlet Allocation was proposed by Blei et al. (2003). By introducing the Dirichlet prior distribution, a top-down three-layer Bayesian framework was constructed, and it was carried out from document to topic and from topic to words. The topic distribution and word distribution are randomly determined by the Dirichlet priori, and hence they were widely applied. Research shows that it is very effective to extract important attributes of a product or service from review text using the LDA-based topic model (Guo et al., 2017; Tirunillai and Tellis, 2014). Therefore, LDA is used to extract express service attributes from online reviews.

\subsection{Sentiment Analysis}

Sentiment analysis is a method that uses machine learning, natural language processing, relevant computer programming, and other information technologies. It can analyze the sentiment polarity and degree of online reviews posted by users (Prager, 2006). With the popularity of the Internet, network data has become the focus of data analysis, and sentiment analysis technology has become a research hotspot (Agarwal and Mittal, 2017; He et al.,2018). From the perspective of the applicable situation, sentiment analysis can be divided into two categories: coarse-grained sentiment analysis and fine-grained sentiment analysis. Coarse-grained sentiment analysis reflects the positive, neutral, or negative sentiment polarity and sentiment score of the entire document (Yu and Hatzivassiloglou, 2003). However, for a text with an overall sentiment polarity, the sentiment polarity of various attributes of a product or service will not be consistent in most cases (Kim and Hovy, 2004). Therefore, coarsegrained sentiment analysis cannot reflect the sentiment of specific attributes. Fine-grained sentiment analysis needs to be adopted for mining the sentiment of specific attributes. 
Fine-grained sentiment analysis is based on the service or product attributes (Chintagunta et al., 2010). By extracting the keywords of each attribute and the corresponding sentiment words, customers' preference for various attributes can be found. Therefore, fine-grained sentiment analysis is also called opinion mining based on attributes (Turney, 2002). The extraction of keywords of an attribute and the corresponding sentiment words as a key issue in fine-grained sentiment analysis has been the focus of many researchers. Hu and Liu proposed a dictionary-based method for the extraction of sentiment words (Hu and Liu, 2004). Cruz et al. (2013) incorporated a domain feature into sentiment word extraction, which improved the accuracy of sentiment word extraction. Lau et al. (2014) constructed a domain ontology, which combines keywords with sentiment words and obtains a higher classification accuracy.

\section{METHODOLOGY}

This paper constructed a framework for mining express service innovation opportunities, as shown in Figure 1. The framework consists of three parts: data preparation, service attribute extraction, and service innovation opportunity mining. The first part collects the original data and preprocesses the data to obtain a set of valid reviews. The second part extracts the keywords of service attributes from the valid reviews by LDA-based topic modeling. The third part mines and analyzes the service innovation opportunities by evaluating the customer satisfaction and attribute importance.

\subsection{Data Preparation}

The preparation of data includes data collection and data preprocessing. Firstly, a website with highquality online reviews and reliable content is selected, and the beginning time and ending time of the data source are set. Then, a crawler program is written to download the online reviews. Finally, the original data is preprocessed by cleaning, segmentation, part of speech tagging, removing stop words, and so on.

\subsection{Service Attribute Extraction}

The LDA-based topic model is a top-down three-layer Bayesian probability model from document to topic and from topic to words. The high-dimensional word vector space is converted into a lowdimensional implicit topic space. The topic of each document is given as a probability distribution, and the hidden variable 'topic' is treated as the probability distribution of the words. Then, the document is considered to be the probability distribution of the topic. Figure 2 shows the concept of LDA-based topic modeling. It is a typical bag of words model, that is, a document is composed of a series of words and there is no order relationship between the words. In addition, a document can contain multiple topics and each word in the document is generated by one of the topics.

LDA is used to model and train these words, and the parameters can be set and adjusted by expert experience (Bi et al., 2019; Jiang et al., 2016). A set of identifiable topics (service attributes) can be obtained, along with a set of keywords of different probability distributions contained in each topic. Probability indicates the possibility that each word belongs to the topic, which can be used as a standard to judge the keyword. Based on the topic-word distribution obtained, the express service attribute can be inferred, and the keywords contained in each express service attribute can be selected.

\subsection{Sentiment Analysis}

In order to reflect the customers' satisfaction with different service attributes, fine-grained sentiment analysis is used to analyze the sentimental polarity and degree from the service attribute level. 
Figure 1. Framework of express service innovation opportunity mining

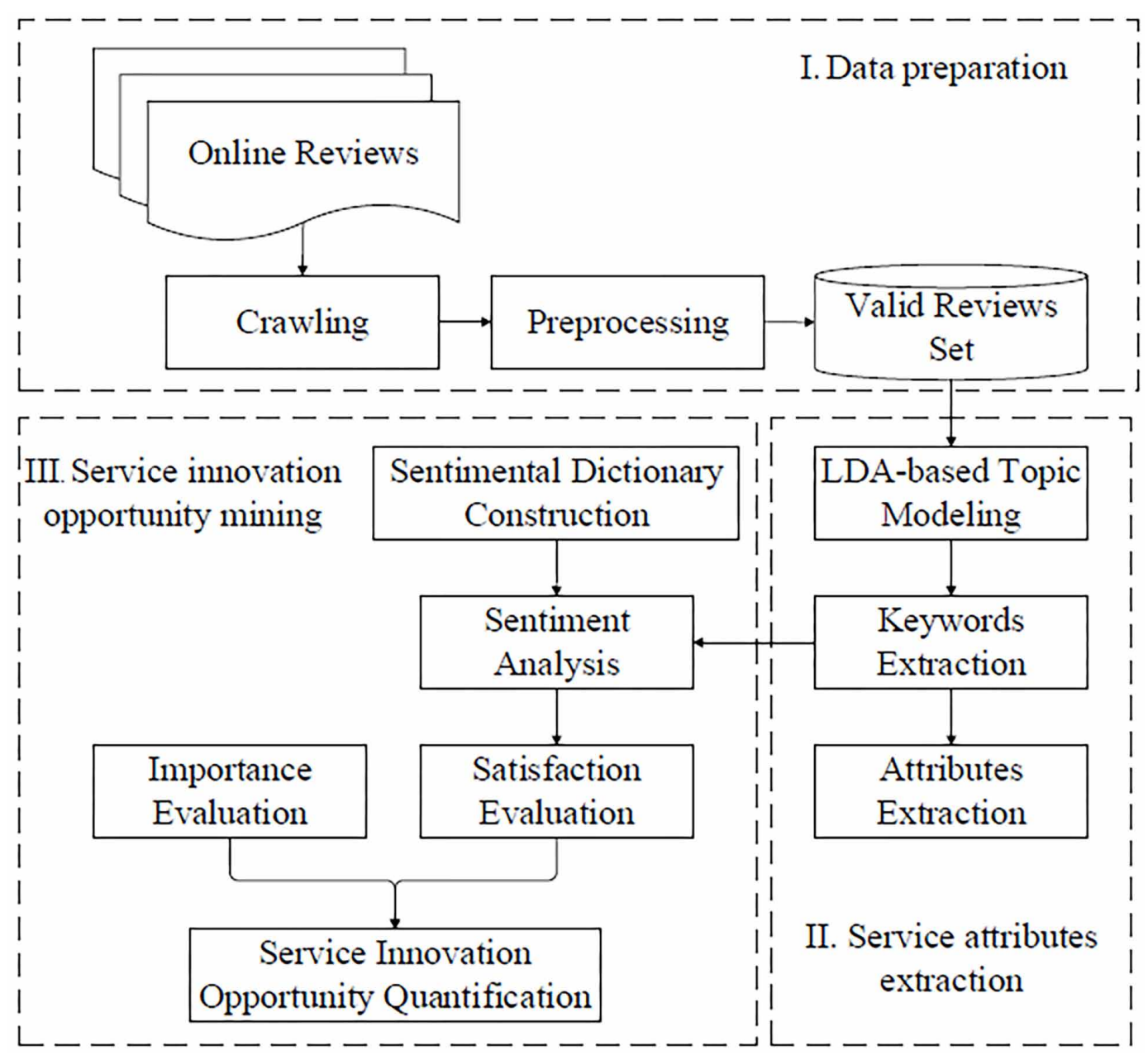

\subsubsection{The Construction of Sentimental Dictionary}

The sentiment analysis method based on sentiment dictionary is adopted. Hownet, which is a Chinese sentiment dictionary including sentiment vocabulary and degree vocabulary, is selected as the basic sentiment dictionary. Since the field adaptability of the basic sentiment dictionary is usually poor, it is also necessary to construct a domain sentiment dictionary to supplement it appropriately.

With regard to the degree words collected by Hownet, there are five intensity levels. The weights are as follows: 2.5 (words such as extreme), 2 (words such as very), 1.5 (words such as more), 1 (no degree words), and 0.5 (words such as insufficiently). Here, 1 indicates the absence of any degree adverbs, greater than 1 indicates enhanced sentiments, and less than 1 indicates weakening sentiments.

\subsubsection{Sentiment Value Calculation}

Each online review can be used as an analysis object. Sentiment analysis unit usually includes sentiment words, degree words and negative words. However, Hownet does not include negative words alone, but combines negative words with sentiment words. Together, they are classified as sentiment words. Therefore, in sentiment analysis, there are only two kinds of cases of sentiment analysis unit. The first is only sentiment words, the second is that sentiment words and degree words exist at the same time. 


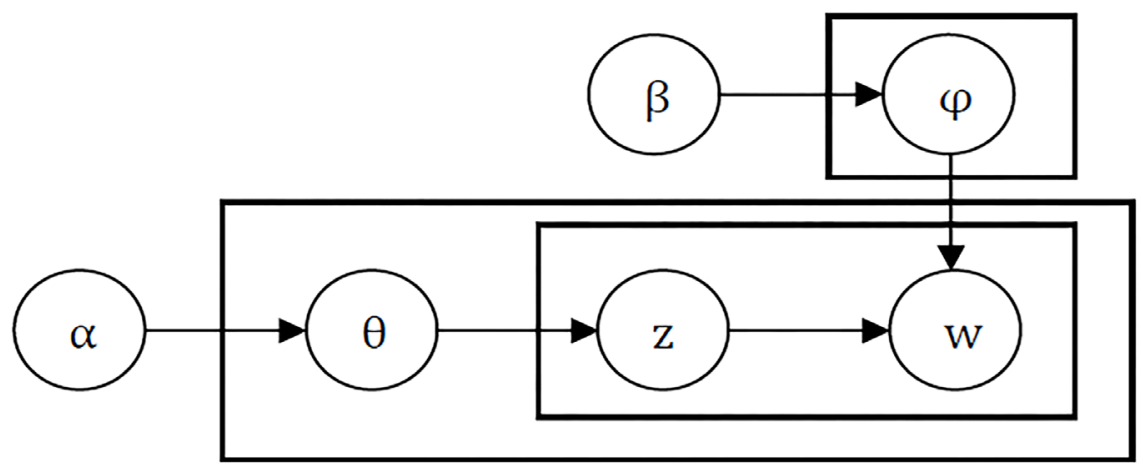

For the above two cases, when there are only sentiment words, the sentiment value of the sentiment analysis unit is equal to the sentiment value of the sentiment words; when the sentiment words and degree words exist at the same time, the sentiment value of the sentiment analysis unit is equal to the score of multiplying the sentiment value of the sentiment words and the weight value of the degree words. With key words as the center, set the sliding window as 3 to match the sentiment words and degree words nearby. The average sentiment value of an attribute can denote the customer satisfaction of this attribute, which can be calculated by Equation (1):

$\operatorname{Sen}\left(A_{j}\right)=\frac{\sum_{t=1}^{T} S\left(w_{i}\right) \times D(a d v)}{N}$

In Equation (1), $\operatorname{Sen}\left(A_{j}\right)$ denotes the sentiment value of the service attribute $A_{j} ; S\left(w_{i}\right)$ denotes the sentiment value of the word $w_{i}$ belonging to the service attribute $A_{j}$ (if it belongs to the positive sentiment, the sentiment value is 1 ; if it belongs to the negative sentiment, the sentiment value is -1 ); $D(a d v)$ denotes the weight of the degree word; $T$ denotes the total number of reviews; and $N$ denotes the number of reviews with sentiment values.

\subsection{Evaluation of Attribute Importance}

Some studies have pointed out that the importance of attributes represented by 'word frequency' is basically consistent with the results perceived by consumers [38]. Based on this conclusion, the 'word frequency' is replaced with the 'number of reviews'. Using the number of reviews that mention words of the service attribute $A_{j}$, the degree of importance of the service attribute is indicated. The importance can be calculated as follows:

$$
\operatorname{Imp}\left(A_{j}\right)=\frac{M}{T}, i=1,2, \ldots, I
$$


In Equation (2), $\operatorname{Imp}\left(A_{j}\right)$ denotes the importance of the service attribute $A_{j} ; M$ denotes the number of reviews referring to the words of the service attribute $A_{j}$; and $T$ denotes the total number of reviews.

\subsection{Quantification of Service Innovation Opportunity}

According to the concept of opportunity algorithm, the importance of service attributes and customers' satisfaction are used to measure the innovation opportunities of this attribute. The more important attribute is considered by customers, the greater the opportunity for innovation. The opportunity value is based on the importance value, combined with the difference between importance and satisfaction. If the difference is greater than 0 , it means that the customer's demand cannot be met, and the score needs to be added. If the difference is less than 0 , it means that the customer's demand is met and the opportunity value is no longer added. The opportunity value is calculated as follows:

Opportunity $\left(A_{j}\right)=\overline{\operatorname{Imp}}\left(A_{j}\right)+\operatorname{Max}\left[\overline{\operatorname{Imp}}\left(A_{j}\right)-\overline{\operatorname{Sen}}\left(A_{j}\right), 0\right]$

In Equation (3), Oppotunity $\left(A_{j}\right)$ denotes the innovation opportunity of service attribute $A_{j}$; $\overline{\operatorname{Imp}}\left(A_{j}\right)$ denotes the normalized importance value of service attribute $A_{j}$, see Equation (4); and $\overline{\operatorname{Sen}}\left(A_{j}\right)$ denotes the normalized sentiment value of service attribute $A_{j}$, see Equation (5):

$$
\begin{aligned}
& \overline{\operatorname{Imp}}\left(A_{j}\right)=\frac{\operatorname{Imp}\left(A_{j}\right)-\operatorname{Im} p_{M i n}}{\operatorname{Im} p_{M a x}-\operatorname{Im} p_{M i n}} \\
& \overline{\operatorname{Sen}}\left(A_{j}\right)=\frac{\operatorname{Sen}\left(A_{j}\right)-\operatorname{Sen}_{M i n}}{\operatorname{Sen}_{M a x}-\operatorname{Sen}_{M i n}}
\end{aligned}
$$

Ulwick (2005) used a scale of 0-10 to divide the opportunity score interval into four categories. Since text mining is used to calculate the score, the score needs to be normalized; therefore, the division interval is changed to $0-1$. The original opportunity interval boundary is narrowed by 10 times and obtained the $0-1$ point innovation opportunity division interval, as shown in Figure 3.

This can be described as follows: when the score is greater than 1.5, it represents an extreme opportunity that cannot be ignored; when the score is between 1.2 and 1.5 , it is described as a 'lowhanging- fruit', which means that it is time to improve and innovate; when the score is between 1 and 1.2, it is still worth considering innovation, especially in a mature market; when the score is less than 1 point, it means that in most markets, these service attributes are not attractive to customers, and the innovation benefits are less.

\section{CASE STUDY}

\subsection{Experiment Data}

The experiment data is obtained from Baidu Koubei (http://koubei.baidu.com). It is a platform of user-generated content aggregation with the theme of business reputation. It has users' real reviews 


\section{Opportunity Score}

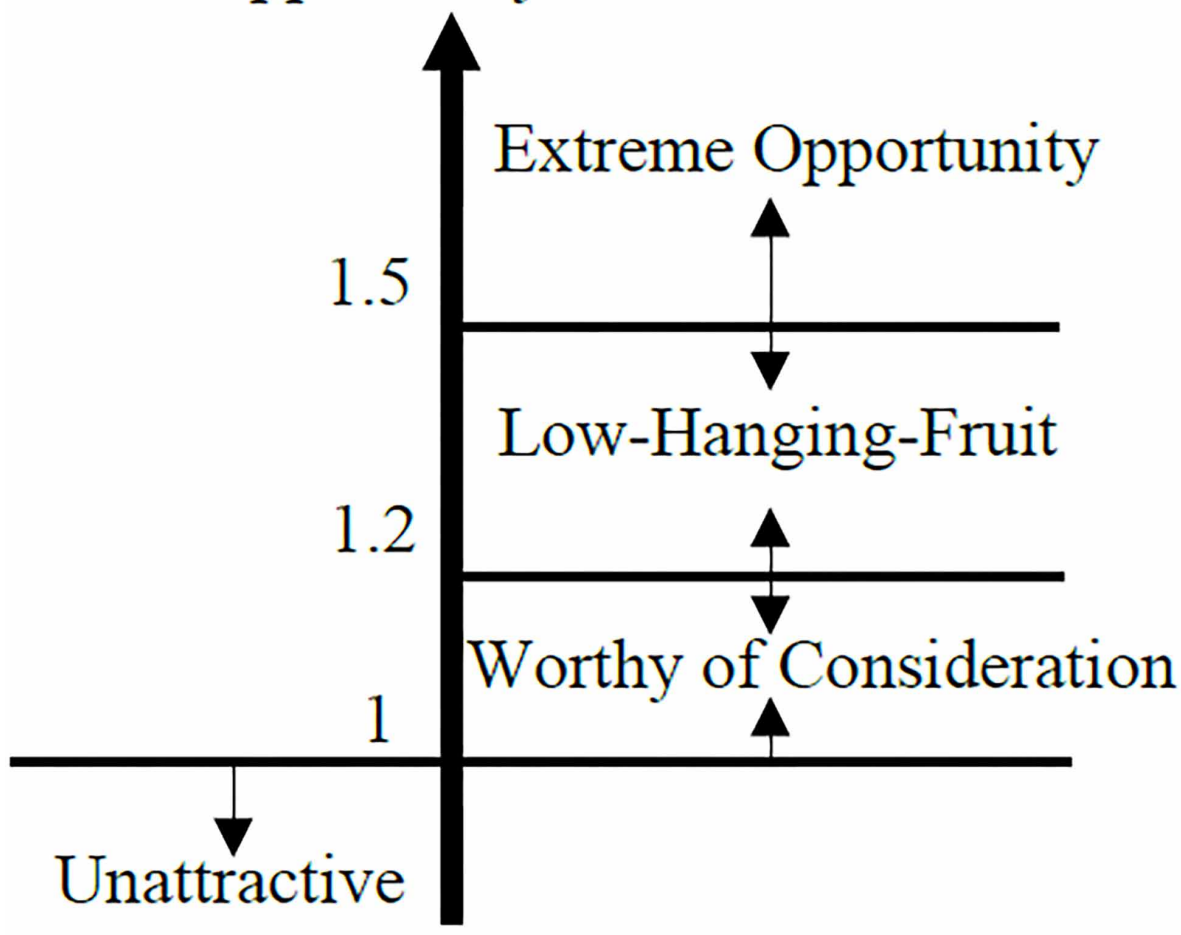

on the Internet word-of-mouth of major merchants. Therefore, in this experiment, Baidu Koubei was selected as the data source.

Considering the express delivery volume, popularity, and number of online reviews, eight express enterprises were selected as the representative enterprises in China, namely STO Express, YTO EXPRESS, ZTO Express, SF Express, YUNDA, EMS, BEST EXPRESS, and TTK Express. The time interval was set from 2015 to 2017 . A crawler was written to download the online reviews of the above eight express enterprises, and 24,441 online reviews were collected.

\subsection{Data Preprocessing}

\subsubsection{Data Cleaning}

In order to reduce the interference of the uncorrelated data on the experiment, some noise data needs to be washed away. By observing the online reviews of express service, the data that should be filtered out mainly includes the following: 1) Repeated reviews. This includes the same reviews given by one user for multiple express enterprises as well as the same reviews given by multiple users for one express enterprise. 2) Short text that does not contain service attribute words. Set filtering rules. For reviews with less than 3 words, if they do not contain attribute words, they are filtered out. Finally, a total of 24,370 valid reviews were obtained to form a set of valid reviews on express service.

\subsubsection{Word Segmentation and Part-of-Speech Tagging}

Because the words in Chinese texts are linked together and not separated by spaces as in English, the Chinese texts need to be partitioned. In addition, the words related to service attributes are mainly 
composed of nouns, verbs and gerunds, while sentiment analysis mainly focuses on adjectives and adverbs. Therefore, it is necessary to perform word segmentation and Part-Of-Speech tagging on the valid reviews. In the experiment, 'jieba,' which is a Python package, was used to carry out the above processing.

\subsection{Extraction of Express Service Attribute}

Based on the results of word segmentation, nouns, verbs and gerunds are extracted from the valid reviews as candidate attribute words. Then, LDA is used to model candidate attribute words. When setting the parameters, three enterprise experts are invited to determine and adjust the number of topics. Based on experience, the number of topics is initially set to 5-20. With the increasing number of topics, the attributes of express service that can be reflected are also increasing. Until the attributes of express service are overlapped and the attribute words begin to disperse, the number of topics is basically determined. Finally, three experts made the judgment and summarized the express service attributes by domain knowledge. Ten attributes have been identified, as shown in Table 1. The "word frequency' indicates the number of keywords included in each service attribute; and the 'number of reviews' indicates the number of reviews belonging to the service attribute.

As shown in Table 1, ten types of service attributes of express delivery service were obtained from the perspective of customers. Here, 'Fee' (A1) indicates whether the fee charged by the express delivery service is reasonable; 'Receiving and sending' (A2) indicates the service performed in the process of receiving or sending; 'Delivery' (A3) refers to the service provided by the express enterprises in the process of delivering packages to customers; 'Signature' (A4) refers to the service provided by the express enterprises when customers sign up for express delivery; 'Customer service' (A5) refers to the manual consulting service provided by the enterprises; 'Service attitude' (A6) indicates the service attitude of employees towards customers when providing services; 'Contact channel' (A7) indicates whether the channels between customers and employees are smooth; 'Goods safety' (A8) indicates whether the goods arrive at customers safely and completely; 'Circulation speed' (A9) indicates the time spent for the entire process; and 'Information tracking' (A10) represents the service that enables customers to query the location information of the express service in real time.

\subsection{Mining Express Service Innovation Opportunity}

\subsubsection{Assessing Customer Satisfaction and Importance of Attributes}

Before the calculation of sentiment value, a domain sentiment dictionary for express service need to be built. The adjectives and negative words in the valid reviews are extracted as candidate words, and then sorted by the TF-IDF weights. Domain sentiment words are collected by manual tagging. Firstly, candidate words are labeled independently by three postgraduates. They are asked to label the sentiment words they think need to retain in one week. When all three people have finished the tagging, they are asked to compare the results. The words that they jointly identified are retained, and the remaining words need to be discussed. Finally, after the three people reach a consensus, the sentiment words collected will be set as domain sentiment dictionary and added to the basic sentiment dictionary. In this experiment, 327 negative sentiment words and 69 positive sentiment words were collected.

The importance and satisfaction were evaluated using Equation (1) and Equation (2). The results are shown in Table 2.

From the evaluation results of importance, it can be seen that customers generally pay attention to 'Customer service' (A5), 'Service attitude' (A6), 'Contact channels' (A7), and 'Delivery' (A3), among which 'Customer service' (A5) is the most important. In addition, 'Service attitude' (A6) and 'Contact channels' (A7) follow closely, sharing the second place in the importance ranking. It can be seen from the top three attributes of importance ranking that customers are more concerned about the services with which they have direct communication and interaction. Understandably, the customers 
Table 1. LDA-based service attributes and words distribution

\begin{tabular}{|l|l|l|l|l|}
\hline Code & \multicolumn{1}{|c|}{ Service Attributes } & \multicolumn{1}{|c|}{ Keywords (in English) } & Word Frequency & Number of reviews \\
\hline A1 & Fee & fee, price... & 6 & 483 \\
\hline A2 & Receiving and sending & receiving, sending... & 6 & 697 \\
\hline A3 & Delivery & delivery, pick up... & 9 & 1770 \\
\hline A4 & Signature & signature, signing... & 3 & 351 \\
\hline A5 & Customer service & customer service staff... & 6 & 2057 \\
\hline A6 & Service attitude & attitude, service attitude... & 3 & 1866 \\
\hline A7 & Contact channel & contact, phone... & 4 & 1874 \\
\hline A8 & Goods safety & package, goods... & 8 & 864 \\
\hline A9 & Circulation speed & speed, efficiency... & 3 & 1334 \\
\hline A10 & Information tracking & message, logistics message ... & 5 & 858 \\
\hline
\end{tabular}

are concerned about 'Delivery' (A3). Because of the development of e-commerce, most customers only participate in the delivery service in the circulation process, that is, to receive the package.

From the evaluation results of satisfaction, it can be seen that the overall sentiment polarity is negative. When the satisfaction score of service attribute is lower, it indicates that the customers' dissatisfaction degree of the service in the overall market is significantly stronger than the satisfaction degree. This may be caused by several factors. The biggest reason is that customers are generally dissatisfied with the service provided by most express enterprises, and the service attributes need to be improved and innovated. Another reason is that when customers encounter unsatisfactory service, they are more willing to share it than when they receive satisfactory service. However, the negative satisfaction scores show that China's express service is in urgent need of improvement and innovation to meet customer demand and improve international competitiveness. From the satisfaction score, it can be seen that in the overall express market in China, the customer satisfaction of 'Customer service' (A5), 'Service attitude' (A6), 'Contact channel' (A7), 'Goods safety' (A8) and 'Information tracking' (A10) is poor, and the innovation degree needs to be analyzed further.

Table 2. Evaluated results of importance and satisfaction

\begin{tabular}{|l|l|l|l|}
\hline \multicolumn{1}{|c|}{ Code } & \multicolumn{1}{c|}{ Service Attributes } & \multicolumn{1}{c|}{ Importance } & \multicolumn{1}{c|}{ Satisfaction } \\
\hline A1 & Fee & 0.020 & -0.014 \\
\hline A2 & Receiving and sending & 0.029 & -0.134 \\
\hline A3 & Delivery & 0.073 & -0.444 \\
\hline A4 & Signature & 0.014 & -0.322 \\
\hline A5 & Customer service & 0.084 & -0.820 \\
\hline A6 & Service attitude & 0.077 & -0.792 \\
\hline A7 & Contact channel & 0.077 & -0.791 \\
\hline A8 & Goods safety & 0.035 & -0.780 \\
\hline A9 & Circulation speed & 0.055 & -0.194 \\
\hline A10 & Information tracking & 0.035 & -0.747 \\
\hline
\end{tabular}


Table 3. Innovation opportunity of express service attributes

\begin{tabular}{|l|l|l|l|l|l|}
\hline \multicolumn{1}{|c|}{ Code } & \multicolumn{1}{|c|}{ Service Attributes } & $\overline{I m p}$ & $\overline{S e n}$ & Opportunity & \multicolumn{1}{|c|}{ Category } \\
\hline A1 & Fee & 0.077 & 1.000 & 0.077 & Unattractive \\
\hline A2 & Receiving and sending & 0.203 & 0.852 & 0.203 & Unattractive \\
\hline A3 & Delivery & 0.832 & 0.467 & 1.197 & Worth of Consideration \\
\hline A4 & Signature & 0.000 & 0.618 & 0.000 & Unattractive \\
\hline A5 & Customer service & 1.000 & 0.000 & 2.000 & Extreme Opportunity \\
\hline A6 & Service attitude & 0.888 & 0.035 & 1.741 & Extreme Opportunity \\
\hline A7 & Contact channel & 0.893 & 0.037 & 1.748 & Extreme Opportunity \\
\hline A8 & Goods safety & 0.301 & 0.050 & 0.551 & Unattractive \\
\hline A9 & Circulation speed & 0.576 & 0.777 & 0.576 & Unattractive \\
\hline A10 & Information tracking & 0.297 & 0.091 & 0.503 & Unattractive \\
\hline
\end{tabular}

\subsubsection{Quantification of Innovation Opportunities of Express Service}

By using Equation (4) and Equation (5), the importance and satisfaction are normalized, and then the innovation opportunities of express service attributes are calculated according to Equation (3), as shown in Table 3.

According to the opportunity interval, 'Customer service' (A5), 'Service attitude' (A6), and 'Contact channel' (A7) were classified as extreme innovation opportunities that could not be ignored. The importance of these three service attributes is very high, but the customer satisfaction is very low. At present, the service level provided by the express market cannot meet the needs of customers. Therefore, these are the three attributes with the greatest innovation opportunities for express enterprises. Through the observation of the three service attributes, it can be seen that customers are more concerned about direct communication with employees of the express service, and the current market service cannot meet the needs of customers. Express enterprises can carry out service innovation in the above aspects and make use of emerging technologies such as the Internet of Things and AI to attract customers and improve market share and competitiveness by meeting customer needs.

In addition, 'Delivery' (A3) is classified as worthy of consideration. In e-commerce logistics, delivery service, which is the part with the most involvement by customers, is likely to become an innovative action with significant benefits. All the remaining six express service attributes scored less than 1 point for innovation opportunities; that is, they were classified as unattractive innovations. From the perspective of service innovation, express delivery enterprises can avoid spending innovative resources on such service attributes. However, from the perspective of customer satisfaction, enterprises should use other analysis tools, such as IPA analysis, to determine the service improvement strategy.

\section{CONCLUSION}

With the rapid development of e-commerce, China's express enterprises are also thriving. At the same time, customer demands for express service are gradually becoming diversified. This paper uses online reviews as data source and tries to explore the direction of express service innovation from the perspective of customers. An LDA-based topic model and fine-grained sentiment analysis 
were adopted to analyze customer feedback (i.e., online reviews). This paper also quantified service innovation opportunities using an opportunity algorithm and built a framework for mining service innovation opportunities. China's express market was taken as an example to carry out a case study. The research found that the direction of express service innovation is related to the communication part in the service. Three types of service attributes that belong to extreme innovation opportunities has been dug out. The results show that combining computer technology with traditional models can effectively provide a direction for service innovation.

The contributions of this paper are as follows: (1) the combination of text mining and opportunity algorithm, which forms a new construction method for opportunity algorithm. (2) From the perspective of customers, the opportunity algorithm is used to explore service innovation opportunities, thereby providing an appropriate quantitative method for identifying service innovation opportunities as well as new ideas for express delivery enterprises to explore the direction of service innovation. Research has proved that computer science can be combined with service innovation to drive service innovation from the perspective of customers.

The limitations and future work of this paper are as follows. (1) Online reviews are used as the data source, but many social media platforms have false reviews such as malicious comments from competitors, which will cause some errors to the data analysis results. At present, the algorithm of identifying false reviews for Chinese is not ideal. However, due to the large number of online reviews, the proportion of false reviews is relatively small, and the error will exist in the acceptable range. In the future, the research on the characteristics and classification of false reviews can be added to improve the accuracy of the work based on online reviews. (2) A general approach is used to evaluate the importance of service attributes, but there are still some scholars exploring more complex methods. In the future, we can consider the importance evaluation of service attributes from the two directions of enterprises and customers, and explore more accurate methods for importance evaluation. 


\section{REFERENCES}

Abualigah, L. M., Khader, A. T., Al-Betar, M. A., \& Alomari, O. A. (2017). Text feature selection with a robust weight scheme and dynamic dimension reduction to text document clustering. Expert Systems with Applications, 84, 24-36. doi:10.1016/j.eswa.2017.05.002

Agarwal, B., \& Mittal, N. (2017). Machine learning approach for sentiment analysis. Prominent Feature Extraction for Sentiment Analysis, 21-45. doi:10.4018/978-1-5225-1759-7.ch070

Bi, J. W., Liu, Y., Fan, Z. P., \& Zhang, J. (2019). Wisdom of crowds: Conducting importance-performance analysis (IPA) through online reviews. Tourism Management, 70, 460-478. doi:10.1016/j.tourman.2018.09.010

Blei, D. M., Ng, A. Y., \& Jordan, M. I. (2003). Latent dirichlet allocation. Journal of Machine Learning Research, 3, 993-1022. doi:10.1162/jmlr.2003.3.4-5.993

Chen, I. S. N., Fung, P. K. O., \& Yuen, S. S. M. (2019). Dynamic capabilities of logistics service providers: Antecedents and performance implications. Asia Pacific Journal of Marketing and Logistics, 31(4), 1058-1075. doi:10.1108/APJML-12-2017-0308

Chintagunta, P. K., Gopinath, S., \& Venkataraman, S. (2010). The effects of online user reviews on movie boxoffice performance: Accounting for sequential rollout and aggregation across local markets. Marketing Science, 29(5), 944-957. doi:10.1287/mksc. 1100.0572

Cruz, F. L., Troyano, J. A., Enríquez, F., Ortega, F. J., \& Vallejo, C. G. (2013). 'Long autonomy or long delay?' The importance of domain in opinion mining. Expert Systems with Applications, 40(8), 3174-3184. doi:10.1016/j. eswa.2012.12.031

Culotta, A., \& Cutler, J. (2016). Mining Brand Perceptions from Twitter Social Networks. Marketing Science, 35(3), 343-362. doi:10.1287/mksc.2015.0968

Faïz, G. (1998). Innovating in reverse: Services and the reverse product cycle. European Journal of Innovation Management, 1(3), 123-138. doi:10.1108/14601069810230207

Feng, Z. (2019). Constructing rural e-commerce logistics model based on ant colony algorithm and artificial intelligence method. Soft Computing, 24(10), 7937-7946. Advance online publication. doi:10.1007/s00500019-04046-8

Groves, R. M. (2006). Nonresponse rates and nonresponse bias in household surveys. Public Opinion Quarterly, 70(5), 646-675. doi:10.1093/poq/nf1033

Guo, Y., Barnes, S. J., \& Jia, Q. (2017). Mining meaning from online ratings and reviews: Tourist satisfaction analysis using latent dirichlet allocation. Tourism Management, 59, 467-483. doi:10.1016/j.tourman.2016.09.009

He, L., Zhang, N., \& Yin, L. (2018). The evaluation for perceived quality of products based on text mining and fuzzy comprehensive evaluation. Electronic Commerce Research, 18(2), 277-289. doi:10.1007/s10660-0189292-0

He, W., Guo, L., Shen, J. C., \& Akula, V. (2016). Social media-based forecasting: A case study of tweets and stock prices in the financial services industry. Journal of Organizational and End User Computing, 31(5), 1486-1515. doi:10.4018/JOEUC.2016040105

Hinterhuber, A. (2013). Can competitive advantage be predicted? Management Decision, 51(4), 795-812. doi:10.1108/00251741311326572

Hu, M. Q., \& Liu, B. (2004). Mining and summarizing customer reviews. Proceedings of ACM SIGKDD international conference on knowledge discovery and data mining.

Jeong, B., Yoon, J., \& Lee, J. (2019). Social media mining for product planning: A product opportunity mining approach based on topic modeling and sentiment analysis. International Journal of Information Management, 48, 280-290. doi:10.1016/j.jinfomgt.2017.09.009

Jiang, H., Qiang, M., \& Lin, P. (2016). Assessment of online public opinions on large infrastructure projects: A case study of the Three Gorges Project in China. Environmental Impact Assessment Review, 61, 38-51. doi:10.1016/j.eiar.2016.06.004 
Killen, C. P., Walker, M., \& Hunt, R. A. (2005). Strategic planning using QFD. International Journal of Quality \& Reliability Management, 22(1), 17-29. doi:10.1108/02656710510572968

Kim, S. M., \& Hovy, E. (2004). Determining the sentiment of opinions. Proceedings of the 20th International Conference on Computational Linguistics.

Kushwaha, N., \& Pant, M. (2018). Link based BPSO for feature selection in big data text clustering. Future Generation Computer Systems, 82, 190-199. doi:10.1016/j.future.2017.12.005

Lau, R. Y. K., Li, C., \& Liao, S. S. Y. (2014). Social analytics: Learning fuzzy product ontologies for aspectoriented sentiment analysis. Decision Support Systems, 65, 80-94. doi:10.1016/j.dss.2014.05.005

Li, M., Shao, S., Ye, Q., Xu, G., \& Huang, G. Q. (2020). Blockchain-enabled logistics finance execution platform for capital-constrained e-commerce retail. Robotics and Computer-integrated Manufacturing, 65, 101962. doi:10.1016/j.rcim.2020.101962

Liu, L., Nie, X., \& Wang, H. (2012). Toward a fuzzy domain sentiment ontology tree for sentiment analysis. In Proceedings of the 5th International congress on image and signal. IEEE. doi:10.1109/CISP.2012.6469930

Liu, W. H., Wang, D., Zhao, X., Si, C., \& Tang, O. (2019). The framework for designing new logistics service product: A multi-case investigation in China. Asia Pacific Journal of Marketing and Logistics, 31(4), 898-924. doi:10.1108/APJML-12-2017-0338

Paton, R. A., \& McLaughlin, S. (2008). The services science and innovation series. European Management Journal, 26(2), 75-76. doi:10.1016/j.emj.2008.03.001

Pournarakis, D. E., Sotiropoulos, D. N., \& Giaglis, G. M. (2017). a computational model for mining consumer perceptions in social media. Decision Support Systems, 93, 98-110. doi:10.1016/j.dss.2016.09.018

Prager, J. (2006). Open-domain question-answering. Foundations and Trends in Information Retrieval, 1(2), 91-231. doi:10.1561/1500000001

Sundbo, J. (2008). Customer-based innovation of knowledge e-services: The importance of after-innovation. International Journal of Services Technology and Management, 9(3/4), 218-233. doi:10.1504/IJSTM.2008.019704

Tirunillai, S., \& Tellis, G. J. (2014). Mining marketing meaning from online chatter: Strategic brand analysis of big data using latent dirichlet allocation. JMR, Journal of Marketing Research, 51(4), 463-479. doi:10.1509/ jmr.12.0106

Turney, P. (2002). Thumbs up or Thumbs down? Semantic orientation applied to unsupervised classification of reviews. Proceedings of the 40th Annual Meeting of the Association for Computational Linguistics.

Ulwick, A. W. (2005). What customer want: Using outcome-driven innovation to create breakthrough products and service. Journal of Product Innovation Management, 23(5), 464-466. doi:10.1111/j.1540-5885.2006.00217.x

Xiao, S., Wei, C. P., \& Dong, M. (2016). Crowd intelligence: Analyzing online product reviews for preference measurement. Information \& Management, 53(2), 169-182. doi:10.1016/j.im.2015.09.010

Xu, Y., Ren, J., Zhang, Y., Zhang, C., Shen, B., \& Zhang, Y. (2020a). Blockchain empowered arbitrable data auditing scheme for network storage as a service. IEEE Transactions on Services Computing, 13(2), 289-300. doi:10.1109/TSC.2019.2953033

Xu, Y., Zhang, C., Wang, G., Qin, Z., \& Zeng, Q. (2020b). A Blockchain-enabled Deduplicatable Data Auditing Mechanism for Network Storage Services. IEEE Transactions on Emerging Topics in Computing, 1. Advance online publication. doi:10.1109/TETC.2020.3005610

Yen, T. M., Chung, Y. C., \& Tsai, C. H. (2007). Business opportunity algorithm for ISO9001: 2000 customer satisfaction management structure. Research Journal of Business Management, 1(1), 1-10. doi:10.3923/ rjbm.2007.1.10

Yu, H., \& Hatzivassiloglou, V. (2003). Towards answering opinion questions: Separating facts from opinions and identifying the polarity of opinion sentences. Proceedings of 2003 Conference on Empirical Methods for Natural Language Processing. doi:10.3115/1119355.1119372 
Zhang, W., Xu, H., \& Wan, W. (2012). Weakness Finder: Find product weakness from Chinese reviews by using aspects based sentiment analysis. Expert Systems with Applications, 39(11), 10283-10291. doi:10.1016/j. eswa.2012.02.166

Ning Zhang received the Ph.D. degree in management from Nanjing University, Nanjing, China. He is an associate Professor with the Business School of Qingdao University, Qingdao, China. His current research interests include business data analysis and user-generate content. He has undertaken several national and provincial projects.

Rui Zhang is a graduate student at Qingdao University, Qingdao, China. Her research interests include internet data analysis and business intelligence.

Zhiliang Pang is a graduate student at Qingdao University, Qingdao, China. His research interests include internet data analysis and business intelligence.

Xue Liu is a graduate student at Qingdao University, Qingdao, China. Her research interests include internet data analysis and business intelligence.

Wenfei Zhao is a graduate student at Qingdao University, Qingdao, China. Her research interests include internet data analysis and business intelligence. 